\title{
Experiência de baixo custo para determinar a forma da superfície de um líquido em rotação usando o smartphone
}

Low-cost experiment to determine the shape of the surface of a rotating liquid using the smartphone

\author{
Eliane Pereira*10
}

${ }^{1}$ Universidade Federal do Sul e Sudeste do Pará, Instituto de Engenharia do Araguaia, 68560-000, Santana do Araguaia, PA, Brasil.

Recebido em 4 de maio de 2021. Revisado em 1 de junho de 2021. Aceito em 5 de junho de 2021.

\begin{abstract}
Neste trabalho, investigamos uma experiência de baixo custo para explorar a física quando uma "folha de água" está em rotação, sob a ação das forças peso e normal. Uma porção de água com corante de alimento vermelho foi colocada em um recipiente que construímos com acrílico transparente no formato de um paralelepípedo, esse recipiente foi acoplado em um fidget spinner que permite o sistema girar em torno de seu eixo vertical. Estudamos a física envolvida nesse problema e calculamos a curva teórica que descreve o comportamento da água em rotação. Verificamos que a curva depende da largura do recipiente e da velocidade angular. Usamos o aplicativo Phyphox livre para fazer as medidas da velocidade angular e podemos comparar a curva teórica com a curva experimental. Para fazer essa comparação, fizemos um applet no software GeoGebra que possibilitou comparar a teoria com uma foto da experiência.
\end{abstract}

Palavras-chave: Ensino de física, líquido em rotação, experimento de baixo custo, smartphone.

In this work, we investigate a low-cost experiment to explore physics when a "leaf of water" is in rotation, under the action of weight and normal forces. A portion of water with red food coloring was placed in a container that we built with transparent acrylic in the shape of a cobblestone, this container was attached to a fidget spinner that allows the system to rotate around its vertical axis. We studied the physics involved in this problem and we calculated the theoretical curve that describes the behavior of the water in rotation. We verify that the curve depends on the width of the container and the angular velocity. We use the free Phyphox app to take angular velocity measurements and we can compare the theoretical curve with the experimental curve. To make this comparison, we made an applet in the GeoGebra software that made it possible to compare the theory with a photo of the experience.

Keywords: Teaching physics, rotating liquid, low cost experiment, smartphone.

\section{Introdução}

A utilização de laboratórios didáticos no ensino de Física é essencial para o entendimento dos fenômenos físicos e dos modelos teóricos, porém os equipamentos necessários para a obtenção de dados normalmente são caros o que dificulta seu vasto uso no ensino médio e até mesmo no ensino superior. Por essa razão, experimentos elaborados com materiais de baixo custo tornaram-se bastante populares. A ação de elaborar esses equipamentos proporciona ao aluno, melhor compreensão dos conceitos teóricos, experiência por tentativa e erro e envolvimento dos discentes no desenvolvimento dos equipamentos científicos. Apesar das vantagens de construir experimentos com materiais de baixo custo, a dificuldade de conseguir medidas precisas com eles torna sua aplicação incompleta. Na tentativa de resolver esse problema, tecnologias aplicadas ao ensino de Física foram desenvolvidas, por exemplo: o software Tracker [1]

\footnotetext{
*Endereço de correspondência: lilausp@gmail.com
}

que permite fazer análise da experiência através de vídeos, emprego de microcontroladores eletrônicos [2] com muitos sensores que podem ser conectados e usados para fazer medidas e o smartphone permite fazer coleta de dados, graças aos seus vários tipos de sensores que o smartphone possui.

O que poderia influenciar na escolha da tecnologia a ser empregada é a conexão entre custo e benefício e obtenção precisa das medidas. Já mencionamos anteriormente três tecnologias, amplamente utilizadas. Qual delas pode ser destacada a mais apropriada? A primeira é os microcontroladores eletrônicos, a segunda é os softwares que fazem vídeo análises e a terceira é o smartphone que já sai de fábrica munido com vários sensores.

Na primeira possibilidade, a dificuldade encontrada em seu uso em grandes proporções é a necessidade de uma noção sobre programação e eletrônica que muitos docentes e discentes não têm. Assim, a necessidade de um conhecimento antecipado dificulta a utilização de microcontroladores e fica restrito a poucas pessoas. 
Na segunda possibilidade, existem softwares livres que fazem vídeo análise como o Tracker, esse programa é de fácil manipulação e possui aplicação em diversas áreas da física [3 6]. Na terceira possibilidade, o smartphone vem mostrando ser muito útil no ensino de física por possuir vários sensores que possibilita medir grandezas da física [7 9], o tipo de sensor do smartphone depende da sua marca, os sensores normalmente encontrados nestes dispositivos são: o giroscópio mede velocidade angular, o luxímetro mede a intensidade luminosa, o barômetro mede a pressão, o acelerômetro mede aceleração, o magnetômetro mede campo magnético, sensor de proximidade, o higrômetro mede umidade do ar, o GPS mede a posição, o microfone e a câmera. Na última possibilidade a limitação encontrada é que ficamos restritos aos números de sensores do smartphone.

Neste artigo, construímos uma experiência de baixo custo para estudar a física envolvida quando colocamos uma "folha de água" em rotação, ou seja, vamos estudar o que acontece quando um recipiente de água gira em torno de um eixo vertical através de seu centro e determinar uma relação entre a forma da superfície da água contida em um recipiente rotativo e sua velocidade angular sob os efeitos do campo gravitacional e da força centrífuga.

A velocidade angular foi obtida através do aplicativo Phyphox [10] que utiliza o giroscópio do smartphone para fazer as medidas. Para poder comparar os dados experimentais com a teoria usamos o software livre Geogebra [11]. Construímos um applet no Geogebra que mostra a curva teórica de uma "folha de água" em rotação e permite ao usuário alterar o valor da velocidade angular. Inserimos uma imagem, obtida do vídeo feito da experiência, no Geogebra que permitiu comparar os valores experimentais com os previstos pelo modelo teórico.

O aplicativo livre Phyphox trouxe mais uma função para o smartphones, esse aplicativo acessa os diversos sensores do smartphone e o transforma em um laboratório portátil de física [10]. Esse computador de bolso, cada vez mais comum entre estudantes do ensino médio e do ensino superior, tornou-se um grande aliado para os professores na sala de aula e nos laboratórios para fazer coletas e análise de dados experimentais e também pode ser uma importante ferramenta no ensino a distância e na Metodologia Ativa de Aprendizagem. O Phyphox também permite o fácil acesso aos dados e controle da experiência de forma remota por outro dispositivo 10 (computador, smartphone e tablet) na mesma rede WIFI do smartphone.

O GeoGebra [11] é um software livre amplamente utilizado para o ensino de matemática e física [12, 13], esse software fornece suporte para várias atividades acadêmicas, por exemplo, resolução de exercícios, criação de applet, simulação, animação, livros dinâmicos, entre outros. O GeoGebra funciona como uma rede social onde os usuários podem acessar applet criados por outros usuários e também compartilhar os seus. Uma importante característica do applet é que ele torna o usuário em um agente ativo facilitando o aprendizado. Vários applets abordando diferentes assuntos podem ser encontrados na referência [1].

\section{O Experimento}

O aparato experimental mostrado na Figura 1 confeccionado com materiais de baixo custo, é composto por duas partes: uma base responsável por fazer o sistema girar e o recipiente onde colocamos a água. Para construir a base giratória do experimento usamos um Fidget Spinner, uma folha de papel cartão do tamanho A4, uma capinha de celular, pedaços de plástico de embalagem, um pedaço de barbante e fita dupla face transparente da marca Scotch para juntar as peças. Primeiramente, fixamos o Fidget Spinner na folha de papel cartão com fita dupla face, em seguida, construímos com pedaços de plástico da embalagem do Spinner (também poderia ser usado garrafa pet), três peças no formato de U e fixamos com fita dupla face nas alças do Spinner (veja a Figura 2). A função destas peças com formato de U é servir de suporte para o barbante e não deixá-lo escorregar. O barbante possui a mesma função que ele tem em um pião, ou seja, colocar o sistema em rotação. Sobre o Fidget Spinner fixamos com fita dupla fase a capinha do celular. A Figura 2 mostra a base giratória montada.

Na segunda parte do aparato experimental usamos duas placa de acrílico (comprimento 19,330 $\pm 0,005 \mathrm{~cm}$, altura $15,415 \pm 0,005 \mathrm{~cm}$ e largura 0,350 $\pm 0,005 \mathrm{~cm}$ ), Silicone Acético transparente, água com corante de alimento vermelho, um pedaço de papelão, supercola e fita preta. Inicialmente, desenhamos um retângulo, nas duas placas de acrílico, de 17,0 $\pm 0,5 \mathrm{~cm}$ de comprimento e 13, $0 \pm 0,5 \mathrm{~cm}$ de altura, também, desenhamos os eixos da coordenada e da abscissa (veja Figura 1). Juntamos as duas placas com o Silicone Acético, o retângulo feito anteriormente serviu para delimitar onde passaríamos o Silicone Acético, deixamos um pequeno orifício para colocar a água com corante vermelho, ela foi injetada

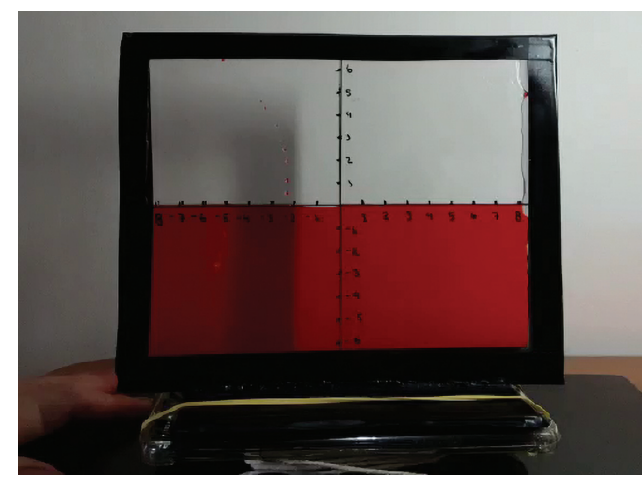

Figura 1: Aparato experimental. 


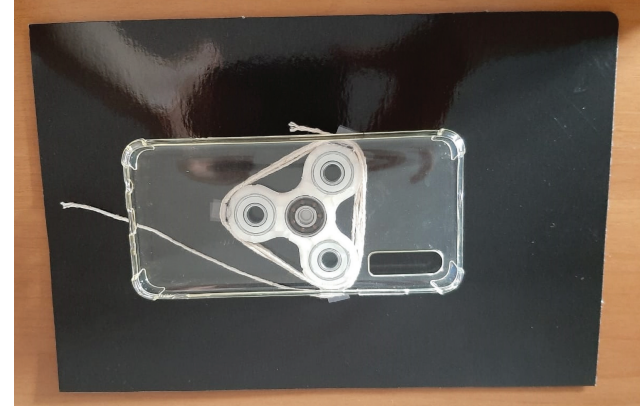

Figura 2: Base giratória.

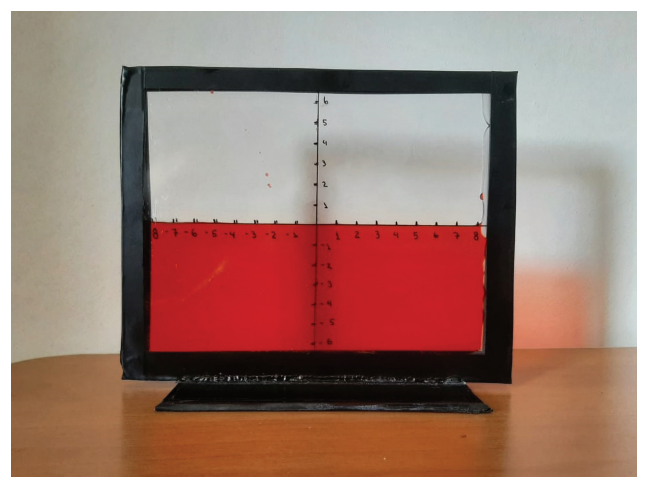

Figura 3: Recipiente com água com corante de alimento vermelho.

com a ajuda de uma seringa, enchemos o recipiente com água até a metade, tampamos com silicone o orifício, para dar acabamento passamos fita preta ao redor do recipiente, finalmente, fixamos o recipiente sobre um pedaço de papelão com supercola (veja a Figura 3). O pedaço de papelão também foi coberto com fita preta. O recipiente construído permite formar uma "folha de água" de comprimento e altura aproximadamente do retângulo desenhado anteriormente e com espessura $0,520 \pm 0,006 \mathrm{~cm}$.

Usamos o aplicativo livre Phyphox para medir a velocidade angular da água, esse aplicativo tem uma vantagem muito útil, ele permite o acesso aos dados e o controle da experiência de forma remota por um notebook ou outros dispositivos [10]. Escolhemos a experiência Giroscópio (taxa de rotação) e no menu, no canto superior direito, escolhemos permitir acesso remoto, confirmamos a mensagem de segurança e surgirá na parte inferior do celular um endereço como http://10 0.0.103.9090 Digitamos esse endereço no navegador do notebook e a partir deste momento podemos controlar e baixar os dados da experiência.

O próximo passo foi encaixar o smartphone na capinha de celular da base giratória, sobre o smartphone colocamos o recipiente com a água, juntamos as duas peças, do aparato experimental, com dois elásticos de látex (veja Figura 1). Colocamos do lado da experiência o notebook que controlou o aplicativo Phyphox, selecionamos a aba

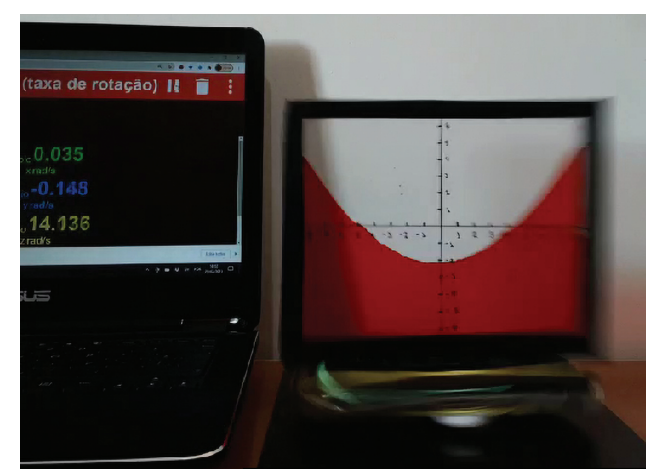

Figura 4: Captura da tela do vídeo quando a "folha de água"está com velocidade angular igual a $14,136 \mathrm{rad} / \mathrm{s}$.

SIMPLES no notebook. O modo SIMPLES mostra na tela os dados da velocidade angular instantânea dos eixos $x, y$ e $z$ do smartphone, aqui estamos interessados somente no valor do eixo $z$. Finalmente, puxamos o barbante para fazer a "folha de água" girar.

O nosso objetivo, neste trabalho, é comparar os dados experimentais com o modelo teórico. Quando colocamos uma "folha de água" em rotação, o modelo teórico prevê que a curva formada pela água é uma parábola, essa parábola depende da velocidade angular medida pelo aplicativo Phyphox. Na próxima seção, veremos os detalhes do modelo teórico.

No software GeoGebra construímos um applet que permite comparar uma foto da experiência com o modelo teórico. Obtemos a foto do vídeo feito da experiência, filmamos a tela do notebook e o experimento como mostra a Figura (4). A ideia é selecionar algumas imagens do vídeo que mostre a curva e a velocidade angular instantânea. O applet tem um controle deslizante que permite ajustar a velocidade angular desejada e além de comparar a foto da experiência com a curva teórica, o applet permite através de caixas de seleção mostrar as forças que atuam em uma porção de água de massa $m$, a área da água deslocada quando o sistema está em rotação, a reta tangente à superfície da água, permite visualizar o ângulo que a reta tangente faz com o eixo $x$ e o ângulo entre a força normal e sua componente vertical. Na próxima seção será explicado a aplicação de cada uma destas funções do applet.

\section{Modelo Teórico}

Realizamos um experimento que visa estabelecer uma relação entre a forma da superfície de um líquido contido em um recipiente rotativo e sua velocidade angular sob os efeitos do campo gravitacional e da força Normal [14, 15. Consideramos os efeitos devidos à tensão superficial desprezíveis.

Na Figura (1), o recipiente de largura $2 a$ está em repouso, ou seja, sua velocidade angular é igual a zero, portanto a superfície do líquido é horizontal. 

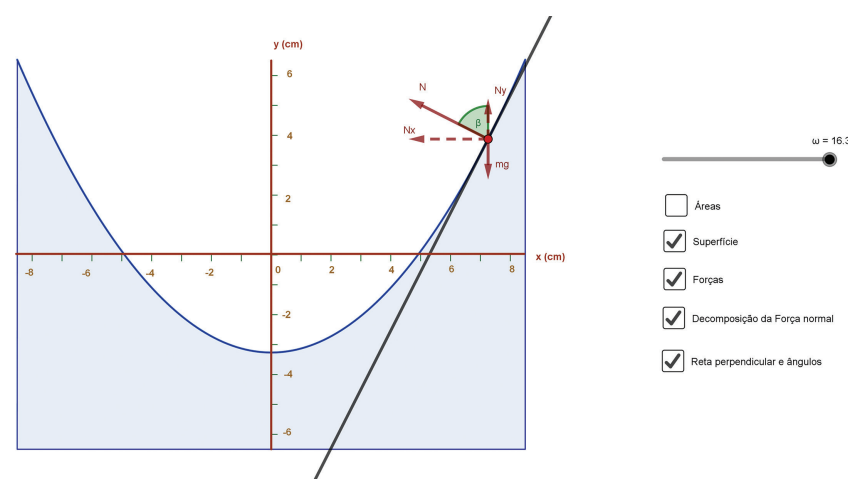

Figura 5: Imagem obtida do applet feito no GeoGebra, quando o líquido está com velocidade angular igual a $16,3 \mathrm{rad} / \mathrm{s}$.

Estabelecemos um sistema de referência não inercial (vinculado ao observador em rotação) de forma que o eixo de rotação seja o eixo y e a superfície do líquido em repouso seja o eixo $x$.

Quando puxamos o barbante que está enrolado no Spinner o sistema entra em rotação e sua velocidade angular varia ao longo do tempo e a superfície do líquido muda de forma. Vamos determinar a equação que descreve a forma da superfície a partir das forças exercidas nas moléculas do fluido.

Do ponto de vista do observador em rotação, as forças que atuam sobre uma partícula de massa $m$ localizada em sua superfície, a uma distância $x$ do eixo de rotação, são o peso $\vec{P}=m g \hat{j}$ e a força normal $\vec{N}$ a reta tangente a superfície da água, como mostra a Figura (5), retirada do animação feita no GeoGebra, quando o líquido está com velocidade angular igual a $\omega=16,3 \mathrm{rad} / \mathrm{s}$.

Decompomos a força normal nas suas componentes vertical $\overrightarrow{N_{y}}$ e horizontal $\vec{N}_{x}$. Quando a velocidade angular é constante, a porção de água de massa $m$ não se desloca, assim $m$ está em equilíbrio vertical, isso implica que

$$
N_{y}=m g,
$$

a porção do líquido de massa $m$ está descrevendo um movimento circular em torno de eixo $y$ e a distância da massa até o eixo $y$ é $x$, a força $\vec{N}_{x}$ é a força centrípeta que é dada pela equação $N_{x}=\frac{m v^{2}}{x}$, no movimento circular uniforme temos $v=\omega x$, combinando as últimas duas equações obtemos $N_{x}=m \omega^{2} x$.

A tangente do ângulo $\beta$ é dado por $\operatorname{tg} \beta=N_{x} / N_{y}$ (veja a Figura 5], substituindo os valores de $N_{x}$ e $N_{y}$, obtemos

$$
\operatorname{tg} \beta=\omega^{2} x / g .
$$

Agora considerando uma variação infinitesimal $d x$ na direção do eixo $x, d y$ na direção do eixo $y$ (veja a Figura 60 e $\alpha$ é ângulo entre a reta tangente a superfície da água e uma reta paralela ao eixo $x$, assim a tangente do ângulo $\alpha$ é dado por:

$$
\operatorname{tg} \alpha=\frac{d y}{d x} .
$$

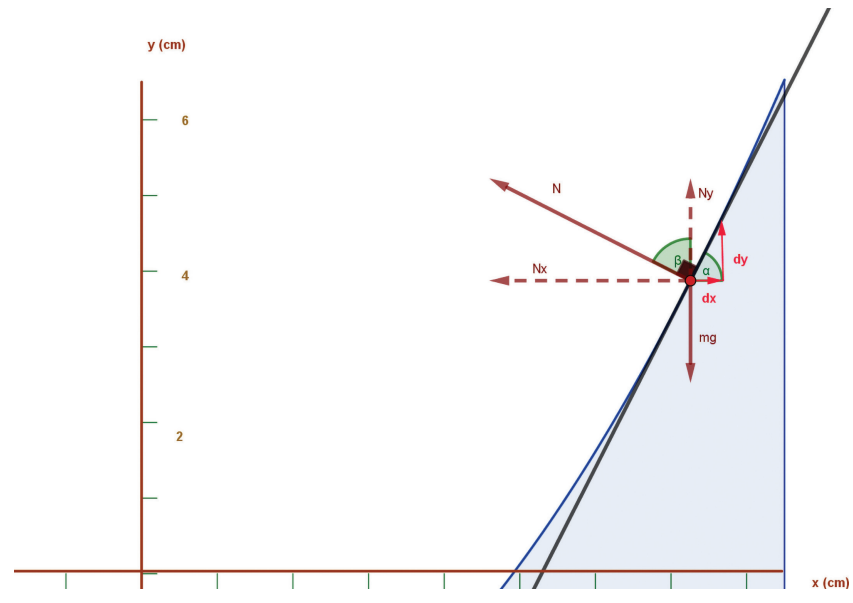

Figura 6: A imagem, obtida do applet feito no GeoGebra, mostra as forças que atuam em porção de água de massa m e os ângulos.

Como a força $\vec{N}$ é normal a reta tangente a superfície da água e observando a Figura (6) concluímos que o ângulo $\beta$ é igual ao ângulo $\alpha$. Portanto as equações (2) e (3) são iguais o que implica:

$$
\frac{d y}{d x}=\frac{\omega^{2} x}{g}
$$

Integrando temos

$$
y=\frac{1}{2} \frac{\omega^{2}}{g} x^{2}+c
$$

que é a equação de uma parábola simétrica em torno do eixo $y, c$ é uma constante de integração.

Para determinar a constante de integração $c$ ou a ordenada do ponto mais baixo da parábola, vamos assumir que o líquido é incompressível. Comparando a situação inicial quando a superfície do fluido é horizontal com a situação final, quando a velocidade angular de rotação é $\omega$. Na situação inicial, a forma da superfície é o segmento da reta $y=0$ entre $-a$ e $a$. Assim a área total deve ser zero no início, quando a "folha do líquido" está em repouso. Quando o sistema está em rotação, observaremos que o líquido afunda na parte próxima ao eixo de rotação e sobe na parte adjacente às paredes do recipiente. Podemos nos convencer disso observando o deslocamento da água na Figura (7) quando a "folha de água" está em rotação com velocidade angular igual a $\omega=16,3$. A área que está abaixo da origem em azul é negativa, a área que está acima da origem em vermelho é positiva e a área total é igual a zero [16, p. 312]. Portanto a integral de $-a$ até $a$ da equação (5) vai ser igual a zero,

$$
\int_{-a}^{a} y d x=0
$$

Resolvendo a integral obtemos a ordenada $c$ do ponto mais baixo da parábola

$$
c=-\frac{1}{6} \frac{\omega^{2} a^{2}}{g} .
$$



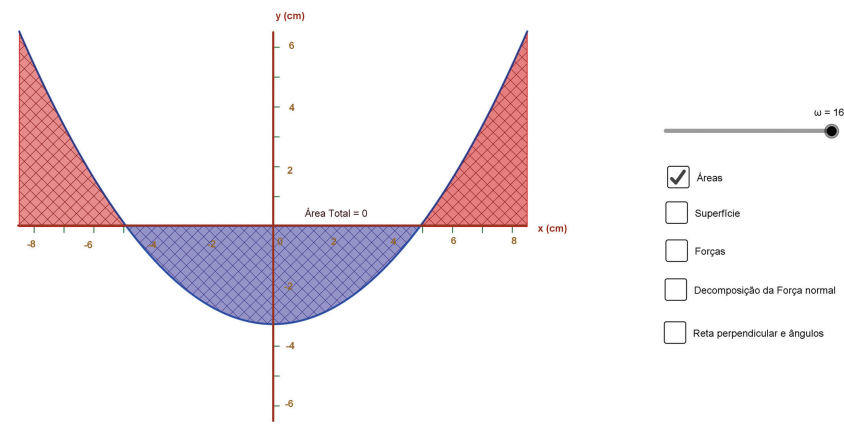

Figura 7: Imagem, obtida do applet feito no GeoGebra, mostra que a área total do líquido deslocado é igual a zero.

A equação da parábola será

$$
y=\frac{\omega^{2}}{2 g}\left(x^{2}-\frac{a^{2}}{3}\right) .
$$

Essa equação é válida em duas situações, a primeira é quando a superfície do líquido é horizontal, ou seja, o sistema está em repouso e a curva da superfície da água é igual à reta $y=0$, a segunda possibilidade é quando o recipiente com água gira, mas o nível da água não atinge a borda superior do recipiente. Para o tamanho do recipiente que construímos, os valores máximo de $\omega$, para que a equação (8) seja válida, é de aproximadamente $\omega=16,3 \mathrm{rad} / \mathrm{s}$, obtemos esse valor isolando $\omega$ da curva (8), adotamos o valor da aceleração da gravidade como sendo $g=9,8 \mathrm{~m} / \mathrm{s}^{2}$, nesta experiência o valor de $a$ foi de $8,5 \mathrm{~cm}$ e os valores máximo de $y$ e $x$ foram $6,5 \mathrm{~cm}$ e $8,5 \mathrm{~cm}$ respectivamente.

\section{Resultados e Discussões}

Para fazer a comparação da teoria com os dados experimentais, capturamos algumas imagens do vídeo feito da experiência, ao fazer a captura da imagem é importante que a "folha de água" esteja o mais paralela possível do celular que filmou a experiência, a imagem contém o aparato experimental e a tela do computador que mostra a velocidade angular obtida pelo aplicativo Phyphox (veja Figura 4), em um editor de imagem selecionamos apenas a parte onde estava o recipiente com água com corante vermelho e inserimos no applet construído no GeoGebra. Usamos a equação (8) para construir a curva teórica no applet. Ajustamos o controle deslizante do applet para velocidade angular indicada na figura, em seguida, ajustamos a imagem até as medidas das ordenada e abscissa da imagem e do applet coincidirem. Consideramos a velocidade angular instantânea constante, pois essa consideração foi importante na dedução da equação 8.

Foram selecionados quatro momentos do vídeo com velocidade angular $\omega$ diferente, as velocidades escolhidas foram 14, $1 \mathrm{rad} / \mathrm{s}, 12,2 \mathrm{rad} / \mathrm{s}, 10,1 \mathrm{rad} / \mathrm{s}$ e $9,1 \mathrm{rad} / \mathrm{s}$, os resultados da comparação da curva experimental com a curva teórica, estão nas Figuras (8), (9), (10) e (11). Nas figuras a curva teórica está em azul. Observe que os dados experimentais estão muito próximos do previsto pela teoria. As pequenas diferenças encontradas entre os dados experimentais e teórico se devem a dois fatores, o primeiro refere-se a dificuldade selecionar uma imagem

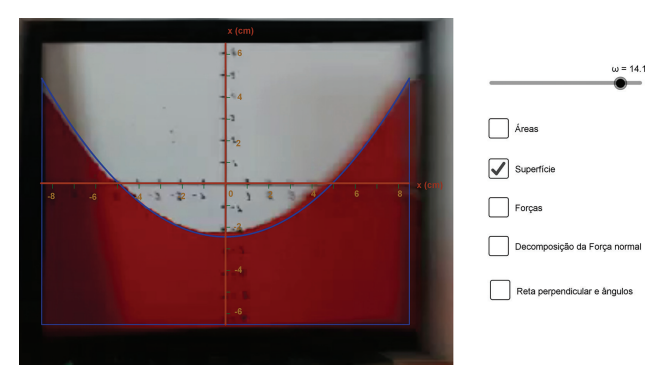

Figura 8: "Folha de água"com velocidade angular igual a $\omega=14,1 \mathrm{rad} / \mathrm{s}$.

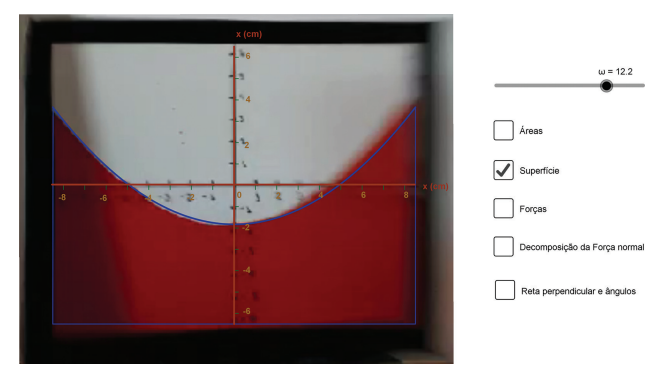

Figura 9: "Folha de água"com velocidade angular igual a $\omega=12,2 \mathrm{rad} / \mathrm{s}$.

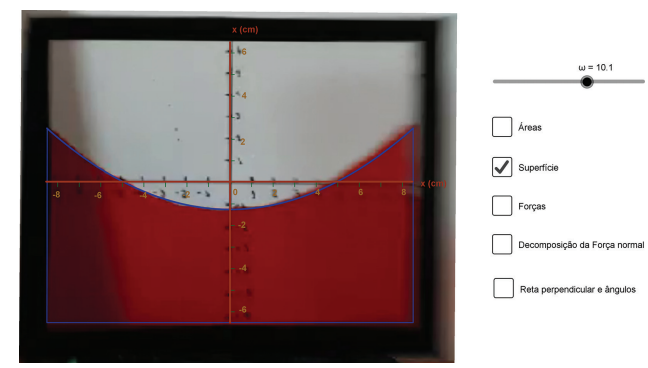

Figura 10: "Folha de água"com velocidade angular igual a $\omega=10,1 \mathrm{rad} / \mathrm{s}$.

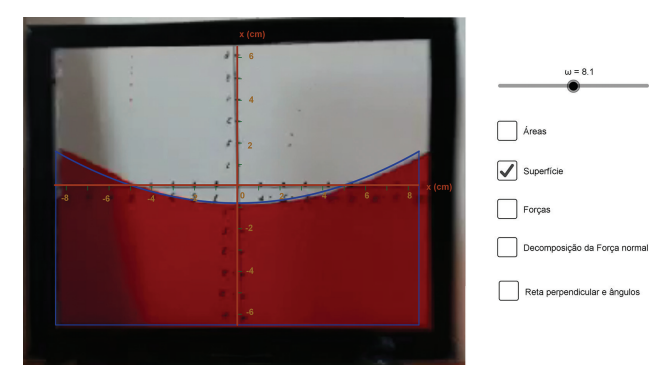

Figura 11: "Folha de água"com velocidade angular igual a $\omega=8,1 \mathrm{rad} / \mathrm{s}$. 
do vídeo onde o recipiente com água esteja paralelo com o smartphone que fez o vídeo, o segundo fator está relacionado ao fato de termos pegado as imagens de um vídeo cuja experiência estava em movimento, isso distorce um pouco a figura.

\section{Conclusões}

O uso de experiências divertidas e atraentes como equipamento de laboratório para abordar conceitos quantitativos têm o potencial de despertar a curiosidade do público aprendiz. Comumente é usado, em sala de aula, o balde de Newton para demonstrar o comportamento de um líquido quando o balde gira em torno de seu eixo vertical, porém essa experiência não é muito atrativa e possibilita apenas uma abordagem qualitativa dos conceitos físicos. Neste trabalho, construímos uma experiência simples, de baixo custo e muito atraente que permite fazer uma abordagem tanto qualitativa, quanto quantitativa dos conceitos de uma "folha de água" em rotação em torno de seu eixo vertical.

O resultado mais importante mostrado foi que é possível comparar as curvas teórica e experimental da "folha de água" em rotação, isso foi possível graças ao aplicativo Phyphox e ao software GeoGebra, ambos são gratuitos. O aplicativo Phyphox fez as medidas da velocidade angular, essa velocidade foi utilizada no applet construído no GeoGebra que possibilitou a comparação de uma foto da experiência com teoria. Os resultados foram bem satisfatórios.

Neste artigo, desenvolvemos uma prática com tecnologia simples, fácil de usar e acessível, por isso acreditamos que a experiência aqui apresentada pode ajudar a incentivar a prática experimental no ensino superior e no ensino médio, proporcionando aos discentes a oportunidade de comprovar a teoria que é apresentada na sala de aula.

\section{Referências}

[1] http://physlets.org/tracker/, acessado em 03/05/2021.

[2] https://www.arduino.cc/en/guide/introduction, acessado em 03/05/2021.

[3] V.L.B. de Jesus, C. Haubrichs, A.L. de Oliveira e D.G.G. Sasaki, European Journal of Physics 39, 025704 (2018).

[4] V.L.B. de Jesus e D.G.G. Sasaki, Revista Brasileira de Ensino de Física 36, 3503 (2014).

[5] A.C. Wrasse, L.P. Etcheverry, G.F. Marranghello e F.S.D. Rocha, Revista Brasileira de Ensino de Física 36, 1501 (2014).

[6] W. Bonventi Jr e N. Aranha, Revista Brasileira de Ensino de Física 37, 2504 (2015)

[7] R.R. Barroso, A.L.D. de Oliveira e V.L.B. de Jesus, Revista Brasileira de Ensino de Física 42, e20200161 (2020).

[8] I. Salinas, M. Monteiro, A.C. Martí e J.A. Monsoriu, The Physics Teacher 58, 569 (2020).
[9] S. Staacks, S. Hütz, H. Heinke e C. Stampfer, Physics Education 53, 045009 (2018).

[10] Your smartphone is a mobile lab, disponível em: https: //phyphox.org/, acessado em 03/05/2021.

[11] https://www.geogebra.org/, acessado em 03/05/2021.

[12] F.F. Barroso, S.A. Carvalho, J.A. Huguenin e A.C. Tort, Revista Brasileira de Ensino de Fisica 40, e2501 (2018)

[13] V. Antohe, arXiv:0905.4430 (2019).

[14] D. Halliday, R. Resnick e J. Walker, Fundamentos de Física (LTC, Rio de Janeiro, 2009), v. 1.

[15] H.M. Nussenzveig, Curso de Física Básica (Edgard Blucher, São Paulo, 2002), v. 1, $4^{\mathrm{a}}$ ed.

[16] H.L. Guidorizzi, Um curso de cálculo (Grupo Gen - LTC, Rio de Janeiro, 2001), v. 1. 